\title{
Sobrecarga em familiares de indivíduos com transtorno obsessivo-compulsivo
}

\section{Burden in families of individuals with obsessive-compulsive disorder}

\author{
Eduardo Batista Soares Neto ${ }^{1}$, João Batista Mendes Teles² ${ }^{2}$ Lúcia Cristina dos Santos Rosa ${ }^{3}$ \\ ${ }^{1}$ Mestrando em Ciências e Saúde pela Universidade Federal do Piauí (UFPI). \\ 2 Professor adjunto de Bioestatística da UFPI. \\ ${ }^{3}$ Professora adjunta da UFPl; orientadora do mestrando.
}

Recebido: 23/11/2009 - Aceito: 31/03/2010

\begin{abstract}
Resumo
Contexto: A presença de sobrecarga em familiares cuidadores resultante do auxílio/cuidado prestado pode comprometer a saúde deles, implicar deterioração de relacionamentos e manutenção ou agravamento do quadro do enfermo. Objetivos: Avaliar graus de sobrecarga, objetiva e subjetiva, de familiares de indivíduos com transtorno obsessivo-compulsivo (TOC) em amostras na rede pública e privada. Métodos: Estudo descritivo-analítico constituído de: caracterização do sujeito com TOC $(\mathrm{N}=30)$ e de seu familiar cuidador $(\mathrm{N}=30)$, em amostras da rede pública $(\mathrm{N}=30)$ e privada $(\mathrm{N}=30)$, por meio de questionários sociodemográficos; confirmação diagnóstica (TOC) com uso do Mini International Neuropsychiatric Interview (MINI); aplicação da Escala de Avaliação da Sobrecarga de Familiares (FBIS-BR). Resultados: Foram detectadas significância estatística entre as amostras na dimensão objetiva, com maiores graus de sobrecarga na amostra da rede pública, e significância na dimensão subjetiva apenas na questão relativa à "realização de tarefas de casa", com maiores graus na amostra da clínica privada. Conclusão: O estudo constatou graus de sobrecarga diferentes em familiares de sujeitos com TOC em tratamento, em amostras na rede pública e privada de assistência em psiquiatria.
\end{abstract}

Soares Neto EB, et al. / Rev Psiq Clín. 2011;38(2):47-52

Palavras-chave: Sobrecarga familiar, transtorno obsessivo-compulsivo, familiar cuidador.

\begin{abstract}
Background: The burden present in family members caretakers that results of the care given, could jeopardize their health, provoke the decay of relationships, as well as maintain or worsen the patient's condition. Objectives: Evaluate the levels of objective and subjective burden in family members of individuals with obsessive-compulsive disorder (OCD) in samples from the public and private network. Methods: This is a descriptive-analytical study that characterizes the individual with $\operatorname{OCD}(\mathrm{N}=30)$ and their family caretakers $(\mathrm{N}=30)$, in samples of public net $(\mathrm{N}=30)$ and private net $(\mathrm{N}=30)$, with use of standardized socio-demographic data questionnaire; diagnosis confirmation (OCD) by using Mini International Neuropsychiatric Interview (MINI); the Family Burden Interview Schedule (FBIS-BR) was applied. Results: A statistical significance has been detected among the samples through an objective point of view, with higher levels of burden in the sample of public net; the significance through a subjective point of view was only perceived regarding "doing housework", with higher levels in the private practice. Discussion: The study has verified different levels of burden in family members of individuals with OCD under treatment in samples from the public and private network of psychiatric assistance.
\end{abstract}

Soares Neto EB, et al. / Rev Psiq Clín. 2011;38(2):47-52

Keywords: Family burden, obsessive-compulsive disorder, family caretaker.

\section{Introdução}

Apesar do crescente interesse em estudos acerca da sobrecarga de cuidadores, destacadamente após o início do processo da reforma do modelo de assistência psiquiátrica, com o envolvimento maior de cuidadores informais no fornecimento de cuidados, verifica-se na literatura carência de estudos investigando graus de sobrecarga de familiares de indivíduos com transtorno obsessivo-compulsivo (TOC).

O processo de desinstitucionalização psiquiátrica com seu modelo comunitário de assistência desencadeou o surgimento de novas necessidades de estruturação do cuidar dos enfermos, novos perfis profissionais e maiores interações com o paciente e o familiar. Nesse contexto, destacam-se, segundo Bandeira et al. ${ }^{1}$, a situação dos serviços comunitários de saúde e o não desenvolvimento de programas de acompanhamento de pacientes na comunidade como razões para um maior envolvimento dos familiares de pacientes psiquiátricos no atributo de principais provedores de cuidados cotidianos ${ }^{1}$.

À família, vem sendo delegado, então, o papel de principal provedora de cuidados e apoio ao paciente, todavia sem preparação, suporte, conhecimento e apoio para a tarefa, resultando em geração de sobrecarga aos cuidadores por causar-lhes frustração pela não efetivação do processo de independência esperado para um indiví- duo adulto $^{1}$ (paciente) e pela não produção de indivíduos saudáveis (física e mentalmente) e preparados para vida e por determinar que aqueles (familiares) abdiquem ou coloquem em segundo plano seus desejos, projetos, necessidades, reorganizando suas vidas ${ }^{2}$ (profissional, familiar etc.) em função, ou com o acréscimo, das necessidades do paciente, em torno das vivências da doença.

O envolvimento familiar na prestação de cuidados e apoio ao seu enfermo pode resultar em consequências negativas para ambos. Pela sobrecarga relacionada à assistência prestada, pela falta de suporte ao seu papel e por estresse, associados aos sintomas da patologia, esses familiares podem ser afetados negativamente ${ }^{3}$.

Relativamente ao indivíduo com TOC - quarto diagnóstico psiquiátrico mais comum ${ }^{4-6}$, encontrando-se entre os cinco transtornos mentais integrantes da lista das patologias implicadas nas maiores taxas de incapacidade do mundo ${ }^{7}$-, registros na literatura têm indicado que cerca de $30 \%$ dos indivíduos não obtêm os resultados almejados com o tratamento, sendo o apoio e o funcionamento familiar fatores extrínsecos ao transtorno que podem interferir nesse resulltado ${ }^{8}$.

A influência familiar pode conduzir positiva ou negativamente a evolução e o tratamento do sujeito com TOC; esta última por meio de mecanismos de acomodação $0^{5,8-11}$ (participação do familiar

Instituição onde o trabalho foi realizado: Universidade Federal do Piauí (UFPI) - Mestrado em Ciências e Saúde.

Endereço para correspondência: Eduardo B. Soares Neto. Rua Vinte e Quatro de Janeiro, 1274, bairro Vermelha - 64018-650 - Teresina, Pl. E-mail: edpsiq@hotmail.com 
nos sintomas, favorecendo a realização da compulsão, terminando por, sem se aperceber, reforçar os sintomas do indivíduo) e de hostilização $0^{5,11}$ (percebida pelo doente em relação a como os seus familiares lidam com seu problema), que podem contribuir para aumentar o grau de desgaste familiar ${ }^{5}$ apesar de os esforços dos familiares serem na tentativa de reduzir o desconforto emocional do paciente e o tempo gasto em rituais. A presença do diagnóstico nos familiares, ou a presença de sintomas ou traços do transtorno neles, pode levá-los a simpatizarem e até a encorajarem comportamentos obsessivo-compulsivos.

Em estudo de revisão, Torresan et al. ${ }^{12}$ observaram que o TOC causa, nos familiares, impacto negativo decorrente de alterações em rotinas, de auxílio a comportamentos e de alterações econômicas. E os familiares às vezes nem se dão conta das alterações em sua qualidade de vida decorrentes da progressiva adaptação aos comportamentos/rituais compulsivos do paciente com TOC. Como resultado da sobrecarga emocional resultante de sentimentos (indulgência, raiva, vergonha, ambivalência) dos familiares para com o portador do TOC, a própria saúde mental deles pode sofrer impacto negativo ${ }^{12}$.

No provimento de cuidados a indivíduos com transtorno mental, existe uma tendência de sobrecarga do cuidado a um único sujeito, que tende a não querer dividir a função com outros indivíduos, apesar de queixar-se da sobrecarga a que se sente submetido. E quando o $\mathrm{faz}$, tende a dividir apenas os aspectos secundários no cuidado ${ }^{13} \mathrm{e}$ a realização de outras funções antes desempenhadas por ele, de modo que fique liberado para dedicar-se ao familiar adoecido; tal ajuda, aceita ou solicitada pelo cuidador, é por este definida e coordenada ${ }^{14}$.

Fatores associados ao cuidado ${ }^{15}$, aos cuidadores ${ }^{15,16}$, à patologia, à relação de dependência e frequência de contato entre cuidador e aquele que recebe cuidados, à falta de adesão ao tratamento, à falta de auxílio ao cuidado por outros familiares, à ausência de informações aos familiares sobre a doença, o tratamento e o manejo prestado pelos profissionais, à adequação de serviços de saúde 2 e à existência de rede de suporte ${ }^{2,16}$ são apontados pela literatura como relacionados ao impacto do cuidar. A presença de comorbidades tem sido identificada como contribuindo para aumento da carga e de custos, e fatores relativos à falta de diagnóstico correto e precoce e ao tratamento inadequado, implicando aumento nos custos ${ }^{17}$.

Aspectos relacionados aos cuidadores como as preocupações com o futuro do familiar adoecido, sentimento de estar aprisionado à situação, sentimentos de responsabilidade como a culpa por não prestarem o cuidado que consideravam que deveriam ser capazes de fornecer e o impacto nas rotinas da família ${ }^{3}$ destacam-se como alguns aspectos da sobrecarga em familiares de indivíduos com transtorno mental.

Loukissa ${ }^{16}$ verificou, em pesquisas avaliadas em estudo de revisão, como consequências negativas da experiência de sobrecarga apresentada pelos familiares, a ocorrência de sintomas de estresse, sentimentos de preocupações com o futuro, medo de reações do familiar doente, tensão, depressão, dificuldades relativas ao sono, alterações no funcionamento familiar, restrição de atividades sociais, medo de estigma, dificuldades financeiras e sentimentos de ansiedade, culpa e raiva e que familiares apresentavam como reação comportamentos como hostilidade e agressividade. Observou ainda que o grau de sobrecarga relacionava-se estatisticamente à gravidade do transtorno mental ${ }^{16}$.

Niederauer et al. ${ }^{4}$ verificaram estudos indicando que no TOC os relacionamentos familiares e sociais são as áreas mais afetadas pelo transtorno ${ }^{4}$.

Alta frequência de envolvimento de familiares nos sintomas dos indivíduos com TOC tem sido verificada em pesquisas, com registros de participação, embora de modo mínimo, de grande percentual dos familiares nas compulsões, ou de modificação de seus comportamentos para adaptar-se aos sintomas dos pacientes, experimentando perturbações em suas vidas - prejuízos em relacionamentos pessoais, problemas financeiros, perda de tempo para lazer - por causa do transtorno ${ }^{11}$, ocorrendo o fenômeno da acomodação em situação de estresse e disfunção familiar?.
Para os serviços de saúde mental, a sobrecarga percebida pelos familiares reveste-se de importância pela possibilidade do desenvolvimento de intervenções mais específicas na família, com a possibilidade de auxílio na reinserção social dos indivíduos acometidos por transtorno mental e de prevenção do surgimento, resultante da sobrecarga, de transtornos psicológicos nos familiares ${ }^{18}$.

No Brasil, poucos estudos acerca da sobrecarga familiar de pacientes psiquiátricos ${ }^{1}$, com a utilização de escalas validadas e adaptadas à realidade do país, são verificados.

A sobrecarga, na literatura, tem sido frequentemente avaliada em duas dimensões: sob a óptica objetiva, distinguindo-se como a consequência observável do cuidar, caracterizando-se pelo excesso de assistência ao paciente, alterações na rotina familiar, supervisão a comportamentos problemáticos, gastos financeiros; e sob a óptica subjetiva, nos aspectos relativos ao grau de incômodo percebido ou avaliado pelo familiar, na assistência ao paciente nas tarefas cotidianas, na lida com comportamentos problemáticos e nas preocupações com o paciente; englobando a reação emocional e o sentimento de estar sendo submetido a uma sobrecarga ${ }^{1,2}$.

Estudos como o de Scazufca et al., investigando a sobrecarga em cuidadores de idosos com depressão ${ }^{19}$, o de Garrido e Menezes, com cuidadores informais de idosos com demência ${ }^{20}$, o de Barroso et al. avaliando a sobrecarga de familiares de indivíduos com transtorno mental - esquizofrenia, transtorno esquizoafetivo e transtorno psicótico agudo de tipo esquizofrênico - em tratamento na rede pública ${ }^{2}$, e o de Lemos et al., estudando cuidadores primários de pacientes com doença de Alzheimer ${ }^{15}$, destacam-se no Brasil, com a utilização de instrumentos validados, no estudo da questão da sobrecarga de familiares.

Partindo da verificação da carência de estudos de sobrecarga em familiares de indivíduos com TOC, e estruturados na hipótese da existência de diferentes graus de sobrecarga conforme a rede assistencial utilizada pelo sujeito com TOC, objetivamos identificar em quais dimensões e em quais aspectos da sobrecarga apresentam-se os maiores graus nas amostras investigadas. Tais investigações podem fornecer subsídios para a identificação de dificuldades no provimento de cuidados pelos familiares cuidadores, de dificuldades nos serviços de atendimento ao portador do transtorno mental e para o desenvolvimento de estratégias de enfrentamento de suas consequências tanto para o portador do transtorno como para o familiar e para a própria rede de assistência.

\section{Métodos}

Para a realização deste estudo descritivo-analítico, foram entrevistados 30 indivíduos com TOC em tratamento na rede privada (consultório de psiquiatra) de Teresina/PI e na rede pública [CAPS (Centro de Atenção Psicossocial) tipo II], em Piripiri/PI (15 de cada rede de atendimento), e 30 familiares (um referente a cada indivíduo com TOC, e por estes indicados como aquele que mais lhe prestava auxílio/assistência no tocante a seu problema de saúde), todos com idade mínima de 18 anos. Foram excluídos os indivíduos com TOC em tratamento de outro transtorno mental registrado em seu prontuário e familiares com transtorno mental em curso.

Foram utilizados: questionários "sociodemográfico e clínico" (construídos para a pesquisa, dirigidos a indivíduos com TOC e ao seu familiar, submetidos a breve estudo piloto sem detecção de dificuldades e com sua inclusão na amostra); a Escala de Avaliação da Sobrecarga dos Familiares - FBIS-BR (para a investigação dos graus de sobrecarga); o Mini International Neuropsychiatric Interview - MINI (para confirmação diagnóstica de TOC e suspeição de comorbidades existentes).

A FBIS-BR, adaptada e validada para o Brasil1,21,22, deriva-se da escala The Family Burden Interview Schedule - Short Form (FBIS/ SF) - desenvolvida por Tessler e Gamache em 19942. É uma escala multifatorial que analisa a sobrecarga familiar, nas dimensões objetiva e subjetiva. Diversamente de outras escalas, foi desenvolvida, em sua origem, para pacientes psiquiátricos com diagnósticos variados, sendo aplicável a uma vasta população de familiares de pacientes ${ }^{1}$. 
O MINI é uma entrevista diagnóstica estruturada, padronizada, psiquiátrica, breve, desenvolvida em 1990 por médicos (psiquiatras e clínicos) nos Estados Unidos e na Europa, para transtornos psiquiátricos do DSM-IV e CID-1023. No presente estudo, utilizou-se a versão MINI 5.0.0/Brazilian version/DSM-IV, Screen e Core, com intuito de confirmação diagnóstica de TOC e suspeição de comorbidades em todos os sujeitos da amostra de TOC. Inicialmente foi aplicada a versão MINI Screen DSM IV-5.0-2002, composta por questões-filtro, para rastreamento de quadros sindrômicos; na presença de positividade para alguma questão-filtro, procedeu-se à aplicação (excluindo-se módulos opcionais) da versão MINI (Core) para a questão positiva.

\section{Considerações éticas}

A participação de todos os sujeitos ocorreu após autorizações institucionais, cumprimento de considerações éticas e obtenção de consentimento livre e esclarecido de todos os participantes.

A aplicação oral dos instrumentos FBIS-BR e MINI pautou-se nas recomendações específicas para sua aplicação.

O uso do MINI ocorreu com a realização do treinamento obrigatório para sua utilização.

Este estudo é parte de pesquisa de mestrado em Ciências e Saúde que obteve aprovação do Comitê de Ética em Pesquisa da Universidade Federal do Piauí, com registro CAAE: 0180.0.045.000-8.

\section{Adversidades e estratégias de condução}

Em quatro ocasiões, não houve manifestação positiva dos indivíduos com TOC em relação à participação na pesquisa (três na rede privada e uma no CAPS; em uma das quais o indivíduo com TOC não tinha familiares residindo na cidade); nessas situações se procedeu ao convite de novos sujeitos.

Todas as entrevistas ocorreram nos locais definidos para a pesquisa; apenas em uma ocasião, na amostra da rede privada, a entrevista do familiar foi realizada em sua residência, por impossibilidade de ele se ausentar de casa (criança pequena e afazeres domésticos).

$\mathrm{Na}$ amostra do CAPS, os indivíduos com TOC foram identificados pelos profissionais do serviço, pois em grande número de prontuários não era feito o registro da codificação do transtorno (CID-10). Todos os diagnósticos foram confirmados com o MINI.

\section{Análise estatística}

Utilizou-se o SPSS 16.0 para o processamento dos dados e os cálculos de médias, frequências e percentuais. Para as análises estatísticas, foram usados: o teste não paramétrico de Mann-Whitney, para verificar diferenças entre os graus de sobrecarga e as amostras das redes, e o teste exato de Fisher, para testar a hipótese de associação entre o tipo de rede de atendimento dos pacientes e os dados sociodemográficos e clínicos.

Para avaliar interações, foi utilizado o modelo linear generalizado, tendo como variável dependente os graus de sobrecarga global objetiva e subjetiva, como fator fixo, a rede de assistência e como cofatores, o gênero e o estado civil do indivíduo com TOC, as comorbidades com o diagnóstico de TOC, a escolaridade e a renda do cuidador.

O poder da amostra, calculado por meio do programa nQuery Advisor 3.0, foi de 95\%.

Em todos os testes trabalhou-se com um nível de significância em $5 \%$.

\section{Resultados}

Como características do sujeito com TOC, observou-se que a média das idades foi de 35,40 anos (desvio-padrão $=13,78$ ). Na amostra total ( $\mathrm{N}=30$ sujeitos com TOC), 56,67\% dos indivíduos eram do gênero feminino, diferenciando-se $(\mathrm{p}=0,025)$ o masculino na amostra de clínica privada (ACP; $\mathrm{N}=15$ sujeitos com TOC) com $66,67 \%$ e o feminino na rede pública/CAPS (ACAPS; $\mathrm{N}=15$ sujeitos com TOC) com $80,00 \%$.
Sessenta por cento eram solteiros na ACP e $60,00 \%$ casados na ACAPS; 46,67\% possuíam ensino superior completo na ACP e $66,00 \%$, fundamental e médio na ACAPS; residiam com o cônjuge e filhos, quando existentes (40,00\% - ACP; 53,33\% - ACAPS) e em família chefiada pelo esposo na ACAPS $(46,67 \%)$ e pelo próprio indivíduo na ACP $(40,00 \%)$.

$\mathrm{Na}$ amostra total, $46,67 \%$ eram de desempregados ou desempenhando apenas atividades no próprio lar, consequentemente sem renda, e tinham como religião a católica $(83,33 \%)$.

Constatou-se significância no tocante à codificação diagnóstica em prontuários ( $\mathrm{p}=0,000$ ), verificando-se sua ausência em $66,67 \%$ deles na ACAPS, enquanto na ACP todos estavam codificados (com $46,67 \%$ dos sujeitos com quadro obsessivo-compulsivo misto). Em todos os casos incluídos no universo amostral, houve a confirmação diagnóstica com o uso do MINI, observando-se suspeição de comorbidades em 40,00\% dos casos (destacando-se: 16,67\% com episódio depressivo maior e $10,00 \%$ com transtorno de ansiedade generalizada).

$\mathrm{O}$ tratamento exclusivamente farmacológico apresentou percentuais de $86,67 \%$ na ACP e $80,00 \%$ na ACAPS, observando-se predomínio $(\mathrm{p}=0,025)$ do uso exclusivo de inibidores seletivos da recaptação de serotonina (ISRS) na ACP em relação à ACAPS.

Os sujeitos apresentaram um tempo médio de latência para obtenção do diagnóstico, desde o início dos primeiros sintomas, de 12,47 anos (desvio-padrão = 8,22) na ACP e de 11,53 (desvio-padrão $=11,44)$ na ACAPS.

O familiar cuidador caracterizou-se por idade média de 45,60 anos (desvio-padrão = 13,26); a amostra total desse grupo $(\mathrm{N}=30$ familiares cuidadores) apresentou destacadamente indivíduo do gênero feminino em ambas as redes de assistência $(80,00 \%)$, católico $(93,33 \%)$, casado $(86,67 \%)$, residindo com o familiar doente $(60,00 \%)$ e cônjuge deste $(36,67 \%)$.

$\mathrm{Na}$ amostra da rede privada (ACP; $\mathrm{N}=15$ familiares cuidadores), destacaram-se o ensino superior completo $(40,00 \%)$ e o médio completo (40,00\%) na ACAPS ( $\mathrm{N}=15$ familiares cuidadores), renda financeira mensal (salário-mínimo atual, R\$465,00) menor que dois salários na ACAPS $(53,34 \%)$ e maior que quatro salários na $\operatorname{ACP}(46,67 \%)$.

Afirmaram não ter outro indivíduo doente sob seus cuidados $76,67 \%$ dos sujeitos. Ainda, 83,33\% informaram não perceber nenhum sintoma físico resultante das atividades que prestava relacionadas ao auxílio/cuidado e 60,00\% informaram não receber auxílio/ orientação no auxílio/cuidado do familiar.

Em relação à consideração familiar de gasto ou não de tempo auxiliando/cuidando, detectou-se uma diferença $(\mathrm{p}=0,002)$ entre as redes. Observou-se que $66,67 \%$ dos sujeitos da ACP consideraram que não gastam tempo cuidando do familiar adoecido, enquanto na ACAPS 46,67\% destacaram dispêndio de menos de duas horas/dia.

Com relação a graus de sobrecarga nos serviços investigados, verificou-se diferença estatisticamente significativa $(p<0,05)$ nas questões discriminadas na tabela 1 .

Os graus de sobrecarga (objetiva e subjetiva) segundo as subescalas, assim como os graus de sobrecarga globais, são listados na tabela 2 .

\section{Discussão}

Os estudos acerca da questão da sobrecarga em familiares ainda são relativamente escassos, com uso de instrumentos validados e adaptados para o Brasilil , todavia têm-se constituído em fonte crescente de interesse não apenas por profissionais, mas também por serviços de saúde. Estudos avaliando os graus de sobrecarga em familiares de indivíduos com TOC em diferentes redes de assistência não são verificados em investigações da literatura.

Além do acréscimo aos conhecimentos existentes, tal estudo pode servir de subsídio e estímulo para novos estudos envolvendo a avaliação de possibilidades de novos recursos em projetos terapêuticos (abordagem familiar), a prevenção de consequências negativas decorrentes de sobrecarga familiar tanto para os indivíduos 
Tabela 1. Graus de sobrecarga conforme questões das subescalas da FBIS-BR* ${ }^{*}$ em familiar cuidador de indivíduos com TOC, segundo rede assistencial

\begin{tabular}{|c|c|c|c|c|c|c|c|}
\hline \multirow{2}{*}{\multicolumn{2}{|c|}{ Subescala }} & \multirow[b]{2}{*}{ Questões } & \multicolumn{2}{|c|}{ Rede privada } & \multicolumn{2}{|c|}{ Rede CAPS*** } & \multirow[b]{2}{*}{$\mathrm{p}$} \\
\hline & & & \begin{tabular}{|c|} 
Grau de \\
sobrecarga
\end{tabular} & $\begin{array}{c}\text { Posto } \\
\text { médio** }\end{array}$ & $\begin{array}{c}\text { Grau de } \\
\text { sobrecarga }\end{array}$ & $\begin{array}{l}\text { Posto } \\
\text { médio** }\end{array}$ & \\
\hline \multirow[t]{3}{*}{ Objetiva } & Assistência na vida cotidiana (A) & $\begin{array}{l}\text { Transporte } \\
\text { Consultas médicas e atividades de tratamento }\end{array}$ & $\begin{array}{l}1,00 \\
1,13 \\
\end{array}$ & $\begin{array}{l}10,00 \\
12,93 \\
\end{array}$ & $\begin{array}{l}2,47 \\
1,53\end{array}$ & $\begin{array}{l}21,00 \\
18,07 \\
\end{array}$ & $\begin{array}{l}0,000 \\
0,046 \\
\end{array}$ \\
\hline & $\begin{array}{l}\begin{array}{l}\text { Supervisão de comportamentos } \\
\text { problemáticos (B) }\end{array} \\
\end{array}$ & Comportamentos desconcertantes & 1,00 & 13,00 & 1,67 & 18,00 & 0,016 \\
\hline & Impacto nas rotinas diárias (D) & $\begin{array}{l}\text { Atrasos ou ausência a compromissos } \\
\text { Alterações das atividades sociais e de lazer } \\
\text { Alterações nos serviços ou rotinas da casa }\end{array}$ & $\begin{array}{l}1,00 \\
1,00 \\
1,00 \\
\end{array}$ & $\begin{array}{l}12,50 \\
13,00 \\
13,50 \\
\end{array}$ & $\begin{array}{l}1,60 \\
1,73 \\
1,53 \\
\end{array}$ & $\begin{array}{l}18,50 \\
18,00 \\
17,50 \\
\end{array}$ & $\begin{array}{l}0,007 \\
0,017 \\
0,035 \\
\end{array}$ \\
\hline Subjetiva & Assistência na vida cotidiana (A) & Realização de tarefas da casa & 2,67 & 5,83 & 1,25 & 2,63 & 0,040 \\
\hline
\end{tabular}

* FBIS-BR (Escala de Avaliação da Sobrecarga dos Familiares - versão brasileira).

** Mann-Whitney test.

*** CAPS (Centro de Atenção Psicossocial) - amostra da rede pública.

Fonte: pesquisa direta.

Tabela 2. Graus de sobrecarga (FBIS-BR*), em familiar cuidador de indivíduos com TOC, segundo rede assistencial

\begin{tabular}{|c|c|c|c|c|c|c|}
\hline \multirow{2}{*}{\multicolumn{2}{|c|}{ Subescalas }} & \multicolumn{2}{|c|}{ Clínica privada } & \multicolumn{2}{|c|}{ CAPS** } & \multirow[b]{2}{*}{$P$} \\
\hline & & $\begin{array}{c}\text { Grau de } \\
\text { sobrecarga }\end{array}$ & Posto médio** & $\begin{array}{c}\text { Grau de } \\
\text { sobrecarga }\end{array}$ & Posto médio** & \\
\hline \multirow[t]{3}{*}{ Objetiva } & Assistência na vida cotidiana (A) & 1,43 & 11,00 & 2,00 & 20,00 & 0,005 \\
\hline & Supervisão de comportamentos problemáticos (B) & 1,12 & 11,73 & 1,57 & 19,27 & 0,013 \\
\hline & Impacto nas rotinas diárias (D) & 1,03 & 11,47 & 1,62 & 19,53 & 0,003 \\
\hline \multicolumn{2}{|c|}{ Sobrecarga global objetiva } & 1,25 & 9,90 & 1,78 & 21,10 & 0,000 \\
\hline \multirow[t]{3}{*}{ Subjetiva } & Assistência na vida cotidiana (A) & 1,58 & 15,63 & 1,37 & 12,70 & 0,304 \\
\hline & Supervisão de comportamentos problemáticos (B) & 2,57 & 8,40 & 2,54 & 8,55 & 0,954 \\
\hline & Preocupação com o paciente (E) & 3,05 & 14,10 & 3,35 & 16,90 & 0,383 \\
\hline \multicolumn{2}{|c|}{ Sobrecarga global subjetiva } & 2,77 & 16,73 & 2,60 & 14,27 & 0,443 \\
\hline
\end{tabular}

* FBIS-BR (Escala de Avaliação da Sobrecarga dos Familiares - versão brasileira).

** Mann-Whitney test.

*** CAPS (Centro de Atenção Psicossocial) - amostra da rede pública.

Fonte: pesquisa direta.

doentes como para o(s) familiar(es), assim como possibilitar (pelo uso de escala padronizada, adaptada e validada para uso no Brasil) a comparabilidade com outros estudos com todos os desdobramentos daí possíveis. Também pode servir de instrumento auxiliar para a identificação de dificuldades no provimento de cuidados pelos familiares cuidadores e de dificuldades nos serviços de atendimento ao portador desse transtorno mental.

Relativamente ao perfil do sujeito com TOC, verificou-se no presente estudo discordância de registros com amostras clínicas que indicam igual prevalência em ambos os sexos ${ }^{5}$ e consonância com relatos de predomínio do gênero feminino em inquéritos populacionais 6 .

Aspectos como a situação ocupacional resultante do acometimento do indivíduo em fases produtivas da vida $\mathrm{e}^{5}$ de comprometimentos pessoais ${ }^{4}$ o quadro clínico composto por formas mistas ${ }^{5} \mathrm{e}$ o fato de residirem com familiares e terem contato diário entre $\mathbf{s i}^{11}$ apresentam-se em concordância com registros na literatura.

A latência para o diagnóstico converge com dados na literatura que indicam período de até 17 anos até a procura e obtenção de tratamento ${ }^{6,12}$.

A presença de depressão como a comorbidade mais frequente encontra-se em concordância com registros na literatura. Essa comorbidade tem sido apontada como se relacionando com gravidade, cronicidade, resposta terapêutica insatisfatória e pior prognóstico ${ }^{24}$, e a existência de sintomas depressivos associados ao TOC tem sido observada como vinculada a alterações negativas mais significativas na qualidade de vida do indivíduo com TOC 4 .

A existência de associação $(\mathrm{p}<0,05)$ entre os tipos de medicações em uso pelos sujeitos com TOC e a rede de assistência utilizada sugere que o tipo de medicação muda em função do local de atendimento, podendo sugerir que a utilização dos inibidores seletivos da recaptação de serotonina sofra influência do aspecto financeiro (possuem custo relativamente alto para a população de menor renda), da disponibilidade de outros fármacos (não pertencentes ao grupo de primeira linha para o tratamento) "gratuitamente" fornecidos pela rede pública aos seus usuários e do grau de conhecimento acerca do problema e do seu tratamento (medicações com melhores evidências de resultados, efeitos colaterais dos fármacos etc.). Aventa-se ainda que tal diferença verificada possa sofrer influência da gravidade dos casos do transtorno - infelizmente não investigada na presente pesquisa -, e uma maior gravidade poderia explicar um maior uso de associações medicamentosas; outra limitação da pesquisa foi a não investigação das dosagens das medicações utilizadas, o que poderia sugerir os níveis de gravidade e iluminar essa análise.

Considerando a carência na literatura de estudos acerca da sobrecarga familiar em indivíduos com TOC, os dados que foram usados para comparação de resultados referem-se principalmente a estudos de sobrecarga de familiares de pacientes psiquiátricos (amostras contendo diagnósticos, principalmente, de esquizofrenia, depressão, transtorno bipolar e quadros demenciais) e de impacto decorrente da presença de sujeito doente na família.

As mulheres destacam-se como as principais cuidadoras $^{2,13,15,16,19-22}$ em estudos que avaliam essa ação, estando implicadas em maior sobrecarga. Ao gênero feminino, histórica, social e culturalmente, associa-se a ação de cuidar. Ao longo da história, a mulher tem vivenciado processos de lutas e conquistas na maioria das vezes resultando tão somente em acréscimo de responsabilidades, na adição de novas tarefas (somando-se o cuidar do lar, da família e dos seus entes ao papel de força financeira ao sustento daquela), sem a valorização devida, em contextos de desigualdades (social, financeira, laboral) de gêneros, de exploração e sobrecarga ${ }^{13}$.

Relacionado à idade média dos cuidadores, verificou-se valor abaixo dos apontados na literatura para cuidadores de diversas patologias, que variaram de 51,40 a 73,2 anos $2,3,15,19-22$. 
A amostra convergiu com estudos que apontam cuidadores casados $^{2,20}$, residindo com o familiar adoecido ${ }^{2,15,19,20}$, com frequência diária de contato com o doente ${ }^{2}$, e com relação ao parentesco, algumas investigações apontam estudos indicando cônjuges ${ }^{16,20}$, embora outros indiquem filhos ${ }^{15,19}$ e pais $23,3,21,22$. Loukissa observou em sua revisão a existência de maior experiência de sobrecarga quando o membro adoentado residia na mesma casa que a família16.

Verificou-se divergência com pesquisas que indicam o ensino fundamental incompleto como mais frequente ${ }^{2}$, desemprego e exercício de atividades do lar ${ }^{20}$, cuidados a outros doentes ${ }^{2}$, presença de queixas de problemas pessoais de saúde pelos cuidadores ${ }^{2}$ e gasto de tempo no cuidar a partir de oito horas por dia ${ }^{15,20}$.

$\mathrm{O}$ aspecto do cuidar de um familiar adoecido, com transtorno mental ou não, como característica constituinte da cultura brasileira ${ }^{2}$ pode constituir-se numa razão para a consideração do familiar cuidador, em nossa amostra, de não ter gasto de tempo com o auxílio/ cuidado.

Os sujeitos desta pesquisa informaram não receber auxílio ou orientações no cuidado a um familiar com TOC, diferentemente do verificado em estudo sobre o impacto em cuidadores de idosos com demência ${ }^{20}$. O aspecto relativo ao sujeito com TOC pode decorrer de sua autonomia verificada na pesquisa, apresentando-se todos em tratamento (com uso de medicações apropriadas) e sem menção de comprometimentos que demandassem a presença de familiar às consultas, além da possível não revelação de seu diagnóstico verdadeiro ao familiar (dados esses não investigados objetivamente e que são assuntos para possíveis novas investigações).

A significância estatística verificada entre as amostras - clínica privada e CAPS -, com relação aos graus de sobrecarga (FBIS-BR) nas questões das subescalas (Tabela 1), indicou que maiores graus de sobrecarga objetiva são verificados na amostra do CAPS. Do mesmo modo, considerando-se os graus de sobrecarga das subescalas, significância estatística foi observada em todas as subescalas objetivas entre as amostras, inclusive com relação à sobrecarga global objetiva (Tabela 2). Refletimos que os resultados possam indicar maior autonomia e talvez maior nível de reserva dos sujeitos com TOC na ACP.

Diversamente, com relação à sobrecarga subjetiva, os maiores graus ocorreram na rede privada, indicando que esses familiares cuidadores apresentariam relativamente maiores sentimentos de incômodo ao prestarem assistência, tendo sido observada diferença estatisticamente significativa apenas na questão "realização de tarefas de casa", $\mathrm{p}=0,040$ (Tabela 1).

A significância observada em relação à sobrecarga global não sofre interação das variáveis gênero e estado civil do indivíduo com TOC e das comorbidades com TOC, nem da escolaridade e da renda do cuidador $(\mathrm{p}>0,05)$.

Estudos com população de baixa renda mostram que certa "perturbação", em virtude do contexto multiproblemático de vida, facilita a melhor aceitação do transtorno mental nesse segmento em relação aos segmentos médios, em que o estigma parece se acentuar ${ }^{25}$.

A verificação de menores graus de sobrecarga no grupo ACP, apesar da afirmação pelos familiares entrevistados desta amostra de que não recebiam ajuda/orientação no cuidado/auxílio (73,33\%), pode refletir a efetividade da farmacoterapia instituída aos sujeitos com TOC desse grupo e/ou maior conhecimento sobre o transtorno (maiores graus de instrução e condição financeira, possibilitando maior acesso ao conhecimento).

Alguns itens relacionados à sobrecarga apresentando maiores graus relativos indicariam a necessidade de investimentos em informação (patologia, cuidados) pelos serviços e profissionais, envolvimento orientado/supervisionado de familiares cuidadores nos tratamentos, além de justificarem a investigação rotineira de graus de sobrecarga nos familiares e de consequências do cuidar, buscando promover a saúde tanto do doente como do familiar cuidador e da família, com todos os seus desdobramentos (gastos, qualidade de vida, relacionamentos etc.), além do aprimoramento dos serviços de saúde.

Especulamos a possibilidade da existência de vieses nesta pesquisa: de seleção (pela natureza e configuração dos locais da coleta da amostra), de aquiescência (pelo uso de instrumento de avaliação disposto em escala) e de gratidão (nas ocasiões em que o indivíduo tivesse realizado consulta anterior com o pesquisador, ou naquelas em que os entrevistados tenham destacado a possibilidade de ter tido a oportunidade de falar mais detalhadamente sobre seus problemas). Ressaltamos ainda os aspectos do tamanho da amostra, das limitações relativas ao método utilizado para a confirmação diagnóstica, da não utilização de instrumento de avaliação da qualidade de vida dos pacientes e de seus familiares e da não comparação dos resultados com um grupo controle de pacientes com outros diagnósticos psiquiátricos como limitações do presente estudo. Destacamos que a sobrecarga observada pode ser resultante da influência da gravidade dos pacientes entrevistados, e não simplesmente do local em que é realizado o tratamento, constituindo a ausência da investigação da gravidade dos pacientes uma limitação existente no presente estudo.

Enfatizamos, entre indicações para novos estudos, a avaliação em indivíduos com TOC "nunca tratados", influência do suporte social, estudos sobre o nível de reserva dos indivíduos com TOC com relação a compartilhamento de problema com familiares e estudos de sobrecarga em outras patologias, com uso de instrumentos adaptados e validados permitindo comparabilidade entre pesquisas, além das implicações do não registro diagnóstico em prontuários no desenho do projeto terapêutico dos enfermos.

\section{Declaração de conflito de interesses}

Declaramos ausência de quaisquer incentivos financeiros na realização desta pesquisa.

\section{Referências}

1. Bandeira M, Calzavara MGP, Varella AAB. Escala de sobrecarga dos familiares de pacientes psiquiátricos: adaptação transcultural para o Brasil (FBIS-BR). J Bras Psiquiatr. 2005;54(3):206-14.

2. Barroso SM, Bandeira M, Nascimento E. Sobrecarga de familiares de pacientes psiquiátricos atendidos na rede pública. Rev Psiq Clín. 2007;34(6):270-7.

3. Rose LE, Mallinson RK, Gerson LD. Mastery, burden, and areas of concern among family caregivers of mentally ill persons. Arch Psychiatr Nursing. 2006;20(1):41-51.

4. Niederauer KG, Braga DT, Souza FP, Meyer E, Cordioli AV. Qualidade de vida em indivíduos com transtorno obsessivo-compulsivo: revisão da literatura. Rev Bras Psiquiatr. 2007;29(3):271-8.

5. Sadock BJ, Sadock VA. Compêndio de psiquiatria: ciência do comportamento e psiquiatria clínica. 9. ed. Porto Alegre: Artmed; 2007, p. 657-65.

6. Torres AR, Lima MCP. Epidemiologia do transtorno obsessivo-compulsivo: uma revisão. Rev Bras Psiquiatr. 2005;27(3):237-42.

7. World Health Organization. As a burden of mental disorders looms large, countries report lack of mental health programmes. Geneva: Press Release WHO/18; 2001. Disponível em: <http://www.who.int/ world-health-day/previous/2001/files/whd2001_press_release_en.pdf $>$. Acesso em: 22 ago. 2009.

8. Ferrão YA, Diniz JB, Lopes AC, Shavitt RG, Greenberg B, Miguel E. Resistência e refratariedade no transtorno obsessivo-compulsivo. Rev Bras Psiquiatr. 2007;29(Suppl 2):S66-76.

9. Calvocoressi L, Lewis B, Harris M, Trufan SJ, Goodman WK, McDougle $\mathrm{CJ}$, et al. Family accommodation in obsessive-compulsive disorder. Am J Psychiatry. 1995;152(3):441-3.

10. Ferrão YA, Aguiar PRDC, Minuzzi L, Grillo R, Lopes R, Rosa R. Características clínicas e história familiar em pacientes ambulatoriais com transtorno obsessivo-compulsivo. Rev Psiquiatr Rio Gd Sul. 2004;26(3):274-9.

11. Steketee G, Van Noppen B. Family approaches to treatment for obsessive compulsive disorder. Rev Bras Psiquiatr. 2003;25(1):43-50.

12. Torresan RC, Smaira SI, Ramos-Cerqueira ATA, Torres AR. Qualidade de vida no transtorno obsessivo-compulsivo: uma revisão. Rev Psiq Clín. 2008;35(1):13-9.

13. Rosa LCS. Transtorno mental e o cuidado na família. São Paulo: Cortez Editora; 2003, 368p.

14. Pegoraro RF, Caldana RHL. Sobrecarga de familiares de usuários de um centro de atenção psicossocial. Psicol Estud. 2006;11(3):569-77. 
15. Lemos ND, Gazzola JM, Ramos LR. Cuidando do paciente com Alzheimer: o impacto da doença no cuidador. Saude Soc. 2006;15(3):170-9.

16. Loukissa AD. Family burden in chronic mental illness: a review of research studies. J Adv Nursing. 1995;21:248-55.

17. Costa AMN. Transtorno afetivo bipolar: carga da doença e custos relacionados. Rev Psiq Clín. 2008;35(3):104-10.

18. Bandeira M, Barroso SM. Sobrecarga das famílias de pacientes psiquiátricos. J Bras Psiquiatr. 2005;54(1):34-46.

19. Scazufca M, Menezes PR, Almeida OP. Caregiver burden in an elderly population with depression in São Paulo, Brazil. Soc Psychiatry Psychiatr Epidemiol. 2002;37:416-22.

20. Garrido R, Menezes PR. Impacto em cuidadores de idosos com demência atendidos em um serviço psicogeriátrico. Rev Saude Publica. 2004;38(6):835-41.
21. Bandeira M, Calzavara MGP, Freitas LC, Barroso SM. Family Burden interview scale for relatives of psychiatric patients (FBIS-BR): reliability study of the Brazilian version [brief report]. Rev Bras Psiquiatr. 2007;29(1):47-50.

22. Bandeira M, Calzavara MGP, Castro I. Estudo de validade da escala de sobrecarga de familiares cuidadores de pacientes psiquiátricos. J Bras Psiquiatr. 2008;57(2):98-104.

23. Medical Outcome System, INC. Jacksonville/Florida U.S.A.: Welcome to the Mini International Neuropsychiatric Interview (M.I.N.I.); 1996-2006. Disponível em: <https://www.medical-outcomes.com/indexSSL.htm>. Acesso em: 20 out. 2008.

24. Petribú K. Comorbidade no transtorno obsessivo-compulsivo. Rev Bras Psiquiatr. 2001;23(Suppl 2):17-20.

25. Duarte LFD. Da vida nervosa nas classes trabalhadoras urbanas. 2. ed Rio de Janeiro: Jorge Zahar; 1993. 